\title{
ORAL CAVITY CHANGES IN THE COURSE OF INFECTIOUS DISEASES DURING CHILDHOOD
}

\author{
Iliyan Todorov ${ }^{1}$, Dimitrichka Bliznakova ${ }^{2}$, Christiana Madjova ${ }^{3}$, Tsvetan Tonchev ${ }^{4}$, \\ Margarita Gospodinova ${ }^{1}$ \\ ${ }^{1}$ Department of Infectious Diseases, Parasitology and Dermatovenereology, \\ Faculty of Medicine \\ ${ }^{2}$ Department of Clinical Medical Sciences, Faculty of Dental Medicine \\ ${ }^{3}$ Department of Conservative Dentistry and Oral pathology, Faculty of Dental Medicine \\ ${ }^{4}$ Department of Oral and Maxillofacial Surgery and Special Imaging Diagnostics, \\ Faculty of Dental Medicine, Medical University of Varna
}

\begin{abstract}
INTRODUCTION: A number of infectious diseases, occurring in childhood take its clinical course with typical changes in the oral cavity. Those changes are pathognomonic sign of the disease or increase the clinical thinking about the concrete disease.

Aim: In the present review we introduce the infectious diseases in childhood that cause different changes in the mouth. In accordance with it we set some tasks: to introduce pathophysiological mechanisms for mucosa damage and its role about diagnosis.

MATERIALS AND METHODS: We based on literature data about that problem. It is discussed in four aspects: diseases with enanthem, changes in the tongue, stomatitis and pharyngitis. Examples and pictures are given.

CONCLUSIONS: Our review presents that the throat inspection in the children with infectious diseases is important stage of clinical examination, should not be missed and the changes have to be recognized in details because of their great diagnostic and differential diagnostic significance.
\end{abstract}

Keywords: rash, enanthema, infection, mouth, oral cavity, childhood

Address for correspondence:

Iliyan Todorov

Department of Infectious Diseases, Parasitology and

Dermatovenereology

Faculty of Medicine

Medical University - Varna

55 Marin Drinov Str.

9002 Varna, Bulgaria

e-mail:ilio_87@abv.bg

Received: November 29, 2015

Accepted: December 14, 2015
The oral cavity is colonized by indigenous flora which inhabits tooth, paradental space and oral mucosa. The oral mucosa implements important functions as covering structures and organs. Because of this together with saliva, gingival secrete and the normal flora, it is the part of the natural immunity in the oral cavity. Variety of microbes may enter in the oral cavity via ingested food or inhaled air. In some cases that microbes start replicating and producing toxins here. It results in edema, vasodilatation and exudation in the affected tissue - for example in the cases of diphtheria. On the other hand - 
in the course of enteroviruses, chicken pox, abdominal fever and etc., the causative agent goes into the bloodstream after its replication in the mouth and regional lymph nodes, where it achieves and damages other organs like liver, kidneys, spleen. In all of the mentioned above situations, by the reason of rich vascularity of the oral mucosa, its innervations and the presence regional lymph nodes typical changes occur which may be seen in simple throat inspection and their role for the diagnostic process is essential.

Enanthem or enanthema are medical definitions meaning a rash on the mucous membranes. Enanthem is presented by different morphology rashes. Hemorrhagic spots - petechiae and ecchymoses are seen very often - in the cases of german measles (named Forscheimer's spots) and infectious mononucleosis (figure 1 and 2) (1).

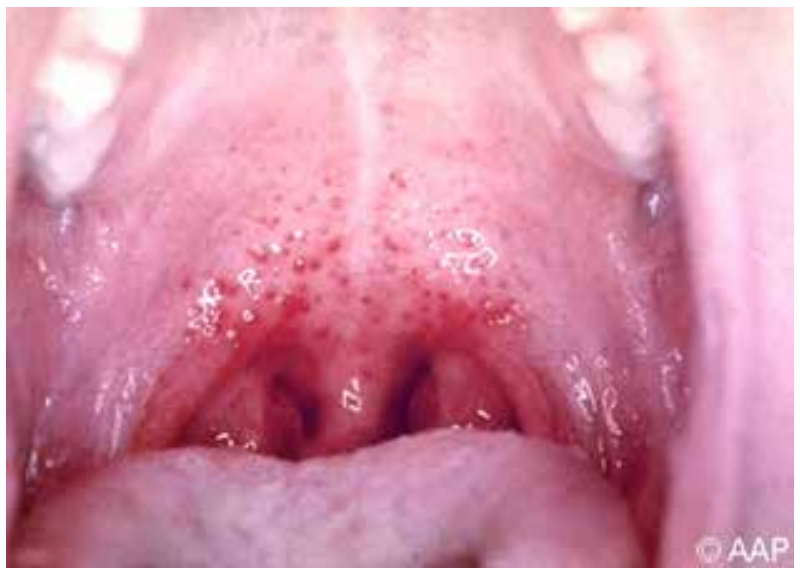

Figure 1. Rubella/German measles/(Hardin MD. University of Iowa and the Centers for Disease Control and Prevention. http://www.lib.uiowa.edu/hardin/Md/ cdc/3185.html)

The typical pathomorphologic change in rickettsioses includes vasculitis. It affects small vessels resulting in hemorrhagic changes. In the cases of Spotted fever we see hemorrhagic lesions on soft palate. It is called Rosenberg's sign.

The hemorrhagic changes are most important in the cases of viral hemorrhagic fevers. From all of know hemorrhagic fevers, Crimean-Congo fever and Hemorrhagic fever with renal involvement are distributed in Bulgaria. They are presented clinically with severe bleeding after the third day of fever occurrence involving gums, which are edematous and blue colored. There is subdental violet strip, which is important for the diagnostic process (figure 3). It has to be distinguished from some ethnic changes in negroid populations where that strip is a normal characteristic in the mouth or in the case of saturnismus. Hemorrhages can be seen on the bucal mucosa, on the soft and hard palate where they seem like trapezium.

In the course of the Febris Papatasii, together with Pick and Tausing's signs, pinpointed hemorrhages appear on the bucal mucosa.

Pathognomonic changes in the mouth occur in the cases of measles - Koplik's spot, which are a prodromic viral enanthem developed two to three days before the measles rash occur. They are characterized as clustered, white lesions on the buccal mucosa (opposite to the lower $1^{\text {st }}$ and $2^{\text {nd }}$ molars). They are

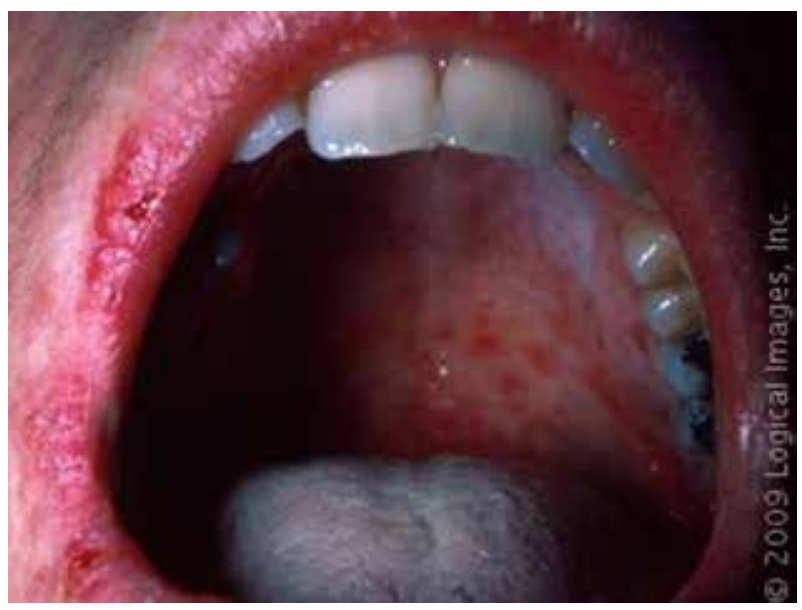

Figure 2. Infectious mononucleosis /Mediscan, Visuals Unlimited, Inc./

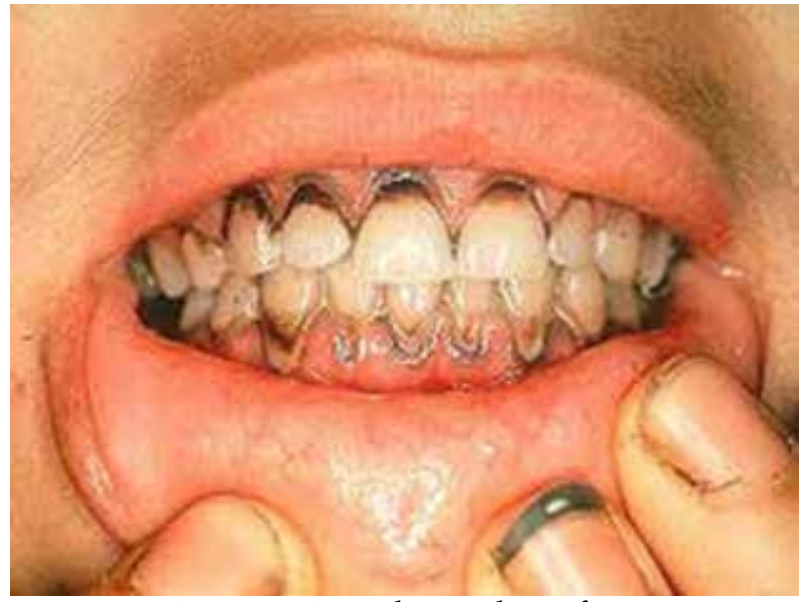

Figure 3. Crimean Congo hemorrhagic fever - patient with subdental hemorrhagic strip /Ball P et al., Infectious diseases - a color guide, Scotland, 1998/. 
Iliyan Todorov, Dimitrichka Bliznakova, Christiana Madjova et al.

described as appearing like "grains of salt on a wet background" and often fade as the maculopapular rash develops (figure 4). They must be distinguished from soor /caused by candida - see below/ especially in infants (1).

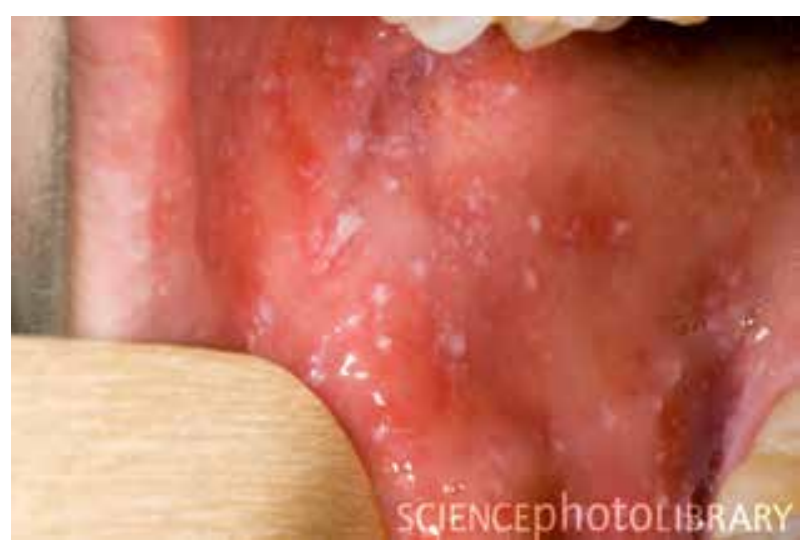

Figure 4. Koplik's spot

Severe immunosuppression and malnutrition of children living in developing countries results in diseases called cancrum oris or noma. It is a rapidly progressive gangrenous infection, due to interaction among Fusobacterium necrophorum and Borrelia vincentii, rarely with Porphyromonas gingivalis, Tannerella forsythia, Treponema denticola etc. The early symptoms of the diseases include formation of violet gingival papula, with central necrosis. It size becomes bigger gradually, affecting lips and chicks. The oral mucosa is edematous, red, painful and after some hours/days necrotic ulcers develop and osteolysis of the face bones can be observed (figure 5).

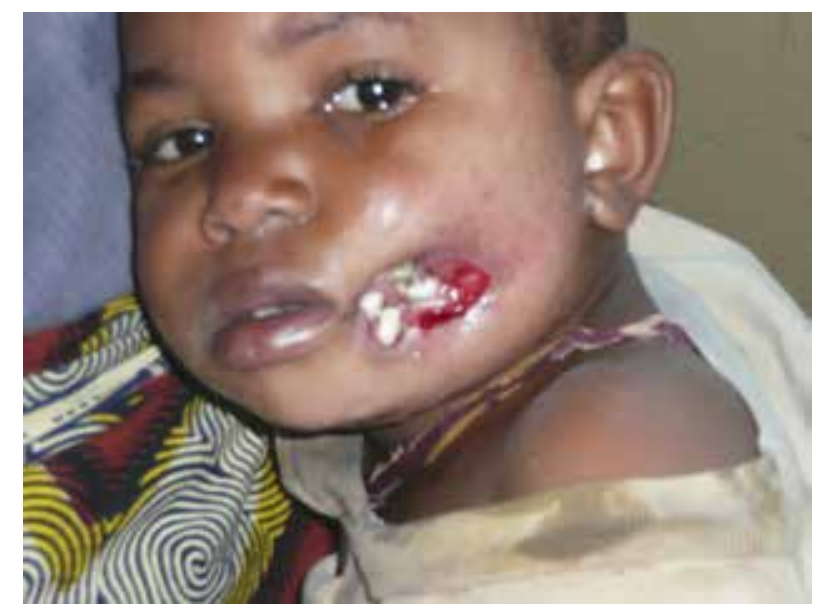

Figure 5. Cancrum oris /noma/ /Ball P et al., Infectious diseases - a color guide, Scotland, 1998/
With the influence of the viral pathogen mucosal lymph follicles become swelled and that changes are most typical about influenza cases - on the soft palate more that 10-20 enlarged papules as a "head of pin” appear - Morozkin's sign.

Exudative rashes in the oral cavity are not seen very often with their typical morphology. Vesicular units evaluate to aphthae and erosions because of the mechanical irritation of the food. It presents the clinical symptoms of the stomatitis and herpangina. Herpangina is viral disease caused by enteroviruses - Coxackie group A, that occurs in the summer, affects children predominantly and oral changes most often are associated with another forms of enteroviral infection - meningitis, diarrhea, exanthema etc. The disease starts acutely, with high level of temperature, sore throat, salivation. On the inspection, more than 10-15 vesicles are seen on the soft palate arranged as a rosary. After some hours they evaluate to shallow erosions which floor is covered by clear exudate. When these changes are associated with skin lesions as vesicles or blisters predominantly on palms of the hands and soles of feet the diagnosis is hand food - mouth disease caused by Coxackie A16 or enterovirus 71 (figure 6).

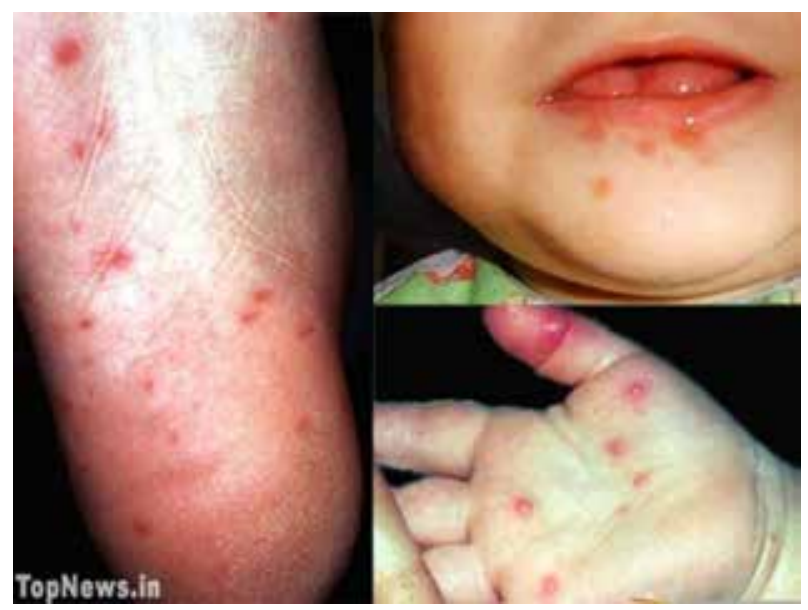

Figure 6. Hand-food-mouth

Enanthema is typical symptom in children with chicken pox. Aphthous ulcers, surrounded by hyperemic halo and covered with white exudate are distributed on the soft and hard palate, gingiva and tongue (figure 7).

In the past, small pox has been a "curse" for the humanity and has taken thousands of victims. Now- 




Figure 7. Oral changes in child with chicken pox I Fitzpatrick's Color Atlas \& Synopsis of Clinical Dermatology, 6th ed. New York: McGraw-Hill, 2009.

Fitzpatrick's Color Atlas and Synopsis of Clinical Dermatology, 6th edition, New York: McGraw - Hill, 2009/.

adays this disease is eradicated thanks to the broadshouldered campaign for vaccination of the people realized by World Health Organization / WHO/ during 1967. In spite of this, symptoms of the small pox have to be known in details because of the possibility for using the causative agent as a biologic weapon. Changes in the oral cavity occur in the beginning of the disease when the mucosa becomes hyperemic diffusely. About the third day of the disease small papula on the soft palate, uvula and palatal arches are revealed. They become vesicles which evaluate to erosions and ulcers covered by fibrinous coating. After superinfection, most often with staphylococcus aureus, septic inflammation achieves in the oral cavity. Very rare mentioned changes occur after vaccination to small pox.

A widespread virus infection that affects all age groups and leads to different kind of changes in the oral cavity is caused by herpes simplex virus (11). Two serotypes of the agent are known- type $1 / \mathrm{HSV} 1 /$ and type 2 /HSV2/. About 1-5 years of age, the child becomes infected with the virus for the first time and after that it stays latent in dorsal root ganglia. In the henceforth, reactivation of the viral replication starts by some provocative factors as immunosuppression, infectious diseases, malnutrition. It leads to specific symptoms of the disease. The children at the breast 6 months aged do not suffer from the HSV1 infection because of the maternal immunity. The prima- ry clinical form of the HSV1 infection is called herpes simplex labialis (figure 8). The patient feels pain and steaming on the affected lip and after some hour small red plaque covered by $10-12$ vesicles filled with yellowish exudate occurs. The period of the disease is about 5 days, after which vesicles become crusts and no more complains are shared. Occurrence of superficial bacterial infection is possible as a complication caused by streptococcus spp. predominantly.

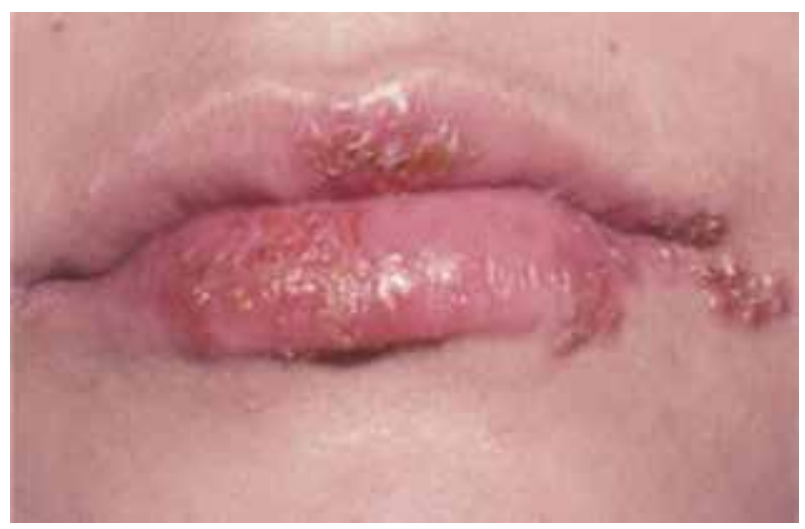

Figure 8. Herpes labialis

Exudative lesions on the mucous membranes are characteristic of the disease impetigo contagiosa, caused by streptococcus serotypes. The disease affects children living in poverty mainly but in older men, a data for pricking on the face or erosions after shaving are reported. The symptoms include formation of small phlyctena on the buccal mucosa, gums and rarely on the tongue which evolve to redness and severely painful erosions. At that moment, diagnosis could be mistaken with other infectious diseases. Coryza and yellow secretion from the nose is the most important distinguishing sign. The mucosa of the nose is most often with erosive changes.

Ulcerative changes in the mouth are typical for the abdominal fever - small erosions on the palatal arches and tonsils reveal, called Duguet's ulcers.

Oral changes on the tongue have important diagnostic role and are common symptoms of different diseases - infectious and non-infectious $(7,10)$. It was said that "the tongue is a mirror of the internal organs" and its changes have to be known in details because of their essential role about diagnosis. In some infectious diseases which take their clinical course with dehydration and intoxication, the tongue be- 
comes furred. Coating is formed by lamination of death epithelial cells, leucocytes, mucus, crystals of cholesterol, food residue and different microorganisms. The furred tongue is the symptom of febrile illnesses, occurs in patient with common cold or with tongue paresis or paralysis /bulbar form of polio, botulism etc./. It occurs also in all cases of gastrointestinal tract infections, but some non-infectious diseases as uremia include that symptom.

Patients with spotted fever have white furred tongue. The disease affects young adults about 15-20 years old and second peak about 40 years of age, rarely newborns. In the course of the disease, paralysis of the nuclei of bulbar cerebrospinal nerves develops resulting in impossibility of the patients to stick out their tongue - Govorov-Gordelie's sign (1).

Furred tongue could be seen in the cases of pestis, because of the severe intoxication. It is cover by thick white coating and seems as "rubbed by chalk".

Typical changes in the tongue of patients with abdominal fever are seen in most of the cases. It its first stage the whole tongue surface except of its apex and edges is covered by white coating. Gradually, after second week the tongue becomes brawn and



Figure 9. Patient with abdominal fever - tongue changes

sometimes it seem with the color of soot (figure 9).

When the scarlet fever occurs, together with the initial triad - fever, sore throat and vomiting, at the time of the mouth inspection, the physician is impressed by the white covered tongue. After 4-5 days from the beginning of the disease, the coating starts to remove from the apex to the root and the li- able mucosa is hyperemic and the papillae are swollen which is compared to a strawberry - ,,strawberry
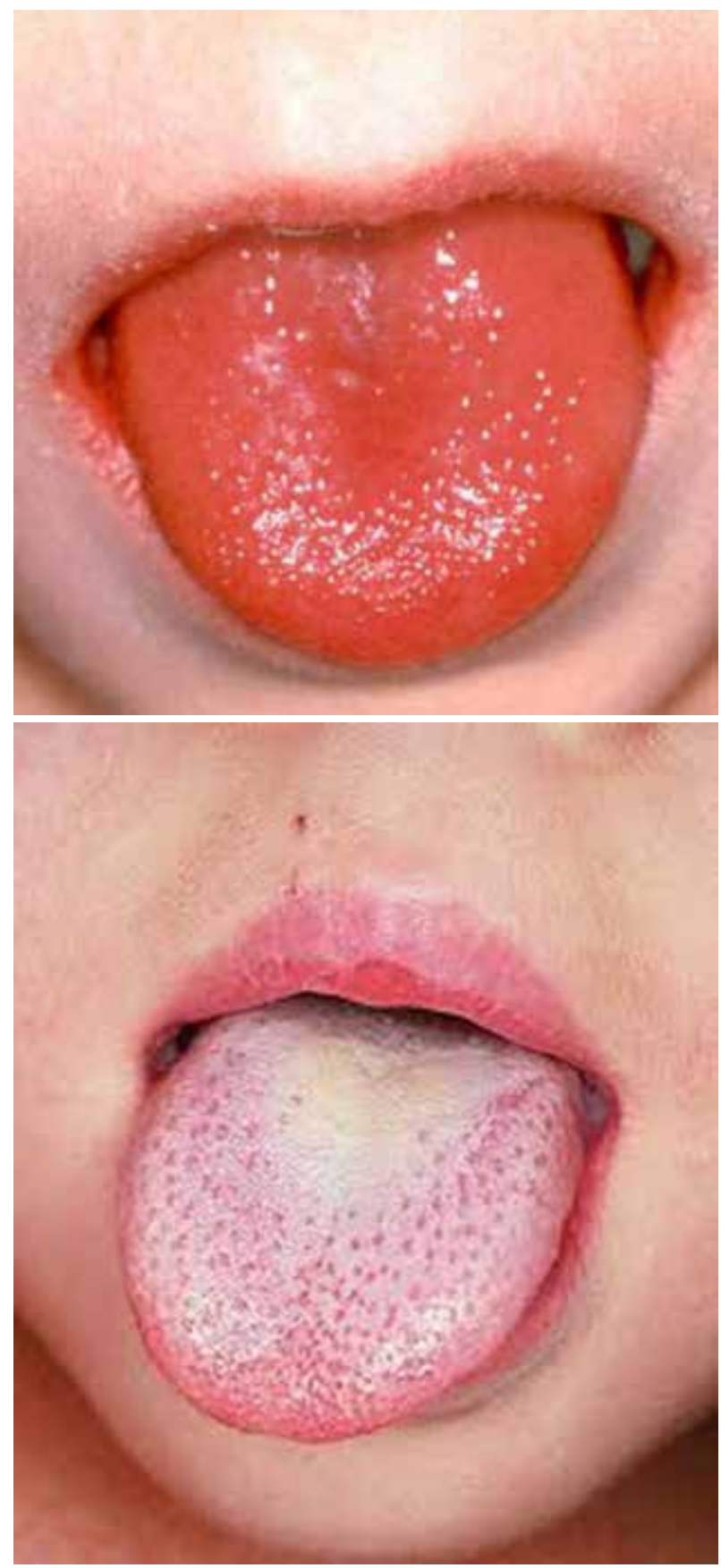

Figure 10. Changes in the tongue in scarlet fever

tongue" (figure 10).

Aphtous changes on the tongue are typical in the patients with chicken pox, enteroviroses. Very often in whooping cough, because of severe cough- 
ing and picking tongue out, ulceration of frenulum lingue develops.

Acquired immune deficiency syndrome /AIDS/ in childhood is a result of intrauterine infection, perinatal period infection and rarely by breastfeeding. One of the visible and important oral changes include the condition called "hairy leukoplakia" (figure 11). It is indicative for AIDS condition, which frequency in children is low. It is associated with $\mathrm{Eb}$ stein-Barr virus /EBV/ infection. On the tongues' edges, unilateral or bilateral, whitish, filiform lesions appear with a corrugative appearance. These lesions cannot be removed by slice. There are no symptoms associated with the lesion although rarely it may be accompanied by steaming, stinging and salivation. The lesions exclusively occur on the lateral surfaces of the tongue, but sometimes on the dorsal surface of the tongue, buccal mucosa, soft palate, pharynx, where "hairy" appearance is absent $(1,2)$.

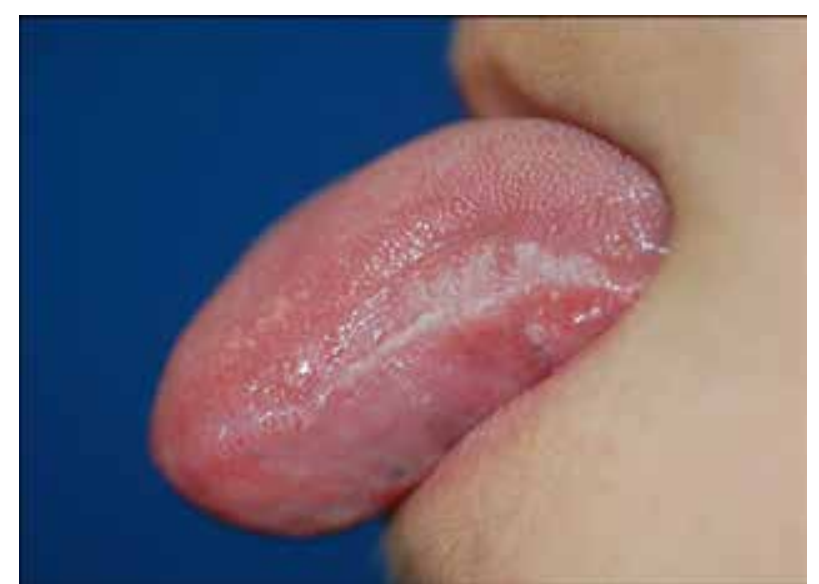

Figure 11. Hairy leukoplakia /Hyun-Ho Cho, M.D., SuHan Kim, M.D., Sang-Hee Seo, M.D. et al., Oral Hairy Leukoplakia Which Occurred as a Presenting Sign of Acute Myeloid Leukemia in a Child, Ann Dermatol. 2010 Feb;22(1):73-76/.

Human immunodeficiency virus /HIV/ associated gingivitis is presented by so called "marginal gingiva" - hyperemic, spontaneously raw, with petechial enanthema gums $(2,7,10)$.

HIV-associated parodontitis is characterized by the destruction of the septum interdentale and fast gingival recession $(2,7,10)$. Rawing of interdenthal papillae and progressive necrosis are typical. In the childhood most often HIV associated ulcero-necrot- ic gingivitis develops. The disease starts acutely with marginal gingiva formation /mentioned above/ and ulcerative and necrotic interdenthal papillae, covered by grey coat (2).

Stomatitis is presented by inflammation of the sublingual or buccal mucosa caused by different microorganisms and their toxins gained to the oral cavity via bloodstream or directly. In most cases it is a symptom of the main disease. There are 5 types of stomatitis - catarrhal, epidemic, aphthous, vesicular, caused by candida $(1,7,10)$.

Catarrhal inflammation on the oral mucosa is often seen in the childhood, as a secondary manifestation of measles, scarlet fever, influenza.

Epidemic stomatitis means stomatitis caused by aphthovirus aphthosae. The other names of the disease are Aphthae epizooticae or aphthous fever. It is zoonotic disease, transmitted by contaminated milk and because of this most often children are affected. The disease starts acutely, with high fever, prostration, myalgia. Severe oral changes are presented steaming, odynophagia, refusal of eating. The oral mucosa is redness and raw when touched. On the buccal mucosis, lips, tongue, gums and tonsils, multitude vesicles develop that evaluate to severe painless aphthae, covered by yellowish, fibrinous exudate. The causative agent disseminates hematogenously which determines the secondary ulcerative lesions on the hands and feet. The prognosis is auspicious and 10-14 days recovery occurs with total epithelization of mucous membranes $(1,7,10)$.

Aphthous enanthema is a symptom of the stomatitis caused by HSV infection. Risk group for development of that disease are children under 7 years of age. To distinguish it from epidemic stomatitis, important fact is that low grade fever is typical and the changes appear in the mouth only. Enanthema occurs on the gingiva mainly as a group of confluent vesicles and never affects tonsils. Severe local pain is the common complaint. The prognosis is favorable $(1,7,8,10,11)$.

The changes mentioned above could be seen in the children with enteroviral infection, but its summer occurrence and associations with another symptoms of enterovirosis as a diarrhea or rashes, support the diagnosis. The oral changes in this case are located on the soft palate and tongue (figure 12). 




Figure 12. Oral candidiasis/C. Halde, Doctorfungus Corporation/

Oral candidiasis /soor, moniliasis/ is caused by Candida albicans and appears in newborns, infants or immunocompromised persons. Often, there are no sign and symptoms of the disease. Complaints include burning feelings in the mouth, painless or changed taste. On the mouth inspection creamy white lesions are seen affecting the tongue, buccal mucosis, gums, tonsils and pharynx. They are slightly raised and look like cheese (figure 13). If the lesions are rubbed slight bleeding occurs. The oral mucosa is inflamed and blushed. The lesions may spread to the esophagus leading to dysphagia.



Figure 13. Enteroviral stomatitis

Tuberculosis in the childhood also may affect the oral cavity. The mean period from bacillary in- oculation and local changes appearance is about 40 days. On the oral mucosa, infiltrative inflammation occurs marked by central shallow ulceration. Its floor is fine granulated seemed as a "mulberry fruit". The lymph nodes are affected in all cases, they are painful, swollen and accreted among each other and to the skin $(7,10)$.

Oral changes appear in the cases of congenital syphilis also. The causative agent crosses the placenta after the 4 month of the pregnancy. The foetus becomes affected in variable aspects depending on the bacterial quantity which was penetrated. After delivery, the baby's face is covered by papulla lesions and its cicatrization leads to radial lesions formation around the lips mainly. That sign is called Parrot's scar. Hutchinson's teeth means morphologic changes in the constantly dentition - frontal upper incisors and the first molar which are compared to a "cask". A furrow which encloses undeveloped yellowish trabeculae is located on their masticatory surface. Syphilitic gumma is located on the soft or hard palate predominantly (10).

The acute respiratory infections remain a common illness among children throughout the world. Airborne infections account for about 7 million outpatient visits annually $(4,5)$. Pharynx and tonsils inflammation is called pharyngitis and tonsillitis (angina) respectively. The special feature in the childhood is small palatal tonsils leading to affection of all adjacent structures - palatoglossal arches, uvula, tonsils and pharynx. Because of this tonsillopharyngitis is more correctly definition in mentioned age group. Classic angina - tonsils inflammation only is typical for young adults and older (4,5,10,13-15).

Pharyngitis in the childhood is caused by streptococcus pyogenes group A in $10-30 \%$ of the cases and affects patients about 5-15 years of age mainly $(4,6,12,15)$. Cases of pharyngitis with different causative agents such as streptococci group $\mathrm{B}$ and $\mathrm{C}$ are rarely seen, approximately in 2-6\%.

The obligatory symptom of scarlet fever is tonsillitis. Together with sore throat, fever and vomiting complaints, typical changes occur in the patients' mouth. The tonsils are swollen, severe hyperemic with redness of the palatoglossal arches, soft palate and uvula - "flaming red catarrh" (figure 14). The angina has various characteristics - catarrhal, follic- 
ular or lacunar. In severe cases, after 2-3 days from the beginning of the disease, tonsils necrosis occurs, which may or may not involve uvula and surrounding structures in the throat $(7,10)$.

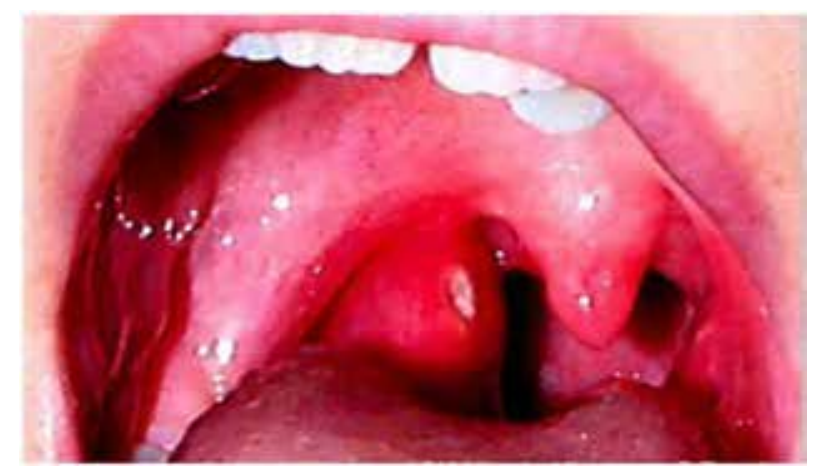

Figure 14. Flaming red catarrh /Vincent MT, Celestin N, Hussain AN: Pharyngitis. American Family Physician. 2004 Mar 15;69(6):1465-70/.

Symptoms of the pharyngitis caused by another types of streptococci (B and C) include complaints such as sore throat, high temperature. In clinical examination severe redness of mucous membranes, resembling to scarlet fever oral changes may be found as a result of catarrhal inflammation $(9,12-15)$.

Arcantobacterium haemolyticus is associated with fever, "flaming throat catarrh", cervical lymphadenitis and sometimes erythematous rash, overwhelming the diagnostic process. It is necessary to be distinguished from scarlet fever.

Staphylococcal angina occurs in children over 5 years old. The symptoms are fever, intoxication, seizures, meningismus. In most cases angina takes the characteristic of lacunar. Rashes seems to those in the scarlet fever are rarely seen $(13,15)$.

Pneumococcal angina has to be distinguished from those with staphylococcal etiology but on base of clinical sign and symptoms it is very harmful (10). Throat smears are helpful.

Diphtheria is an acute infectious disease, airborne infection caused by toxin producing strains of Corynebacterium dyphtheriae, leading to changes in the oral cavity most often, but nose, skin and genitals are affected rarely. Nowadays diphtheria belongs to the group of vaccine preventable diseases and because of this occurs only if the patients are not vaccinated, without revaccination or lack of specific immunity after vaccination. The disease is endemic for developing countries, tropics. Cases are reported in Haiti, Russia, Ukraine $(4,7,9,10,15)$. Sometimes Corynebacterium ulcerans may leads to the similar symptoms, most often if the patient were consumed contaminated, raw milk (10).

Corynebacterium dyphtheriae stays on its entrance and cause fibrinous inflammation forming thick, grey pseudomembranes/coating/ covering the tonsils and spreading to around mucosa. Bacteria produces toxin which spreads into the bloodstream and affects distant organs such as the myocardium, kidneys, central nervous system, inhibiting the protein synthesis of their cells. Most commonly, diphtheria is presented as a throat inflammation which takes its clinical course in different ways: island form - small pointed or bigger grouped coatings on the tonsils; a fused form - the coatings of the tonsils are wide spread oh the whole surface of the tonsil, thick and ceaseless (figure 15); broad spread form - the coating appears on the soft palate, uvula and buccal mucosis; toxic form - swelling of the pharyngeal mucosa and thick pseudomembranes covered the tonsils. Removal of the pseudomembrane reveals a bleeding mucosa. All of these forms occur in combination with fever, severe intoxication, enlargement of the regional lymph nodes because of their swelling /the appearance of the patient's neck resembles to the bull's neck/. The mortality rate is very high.

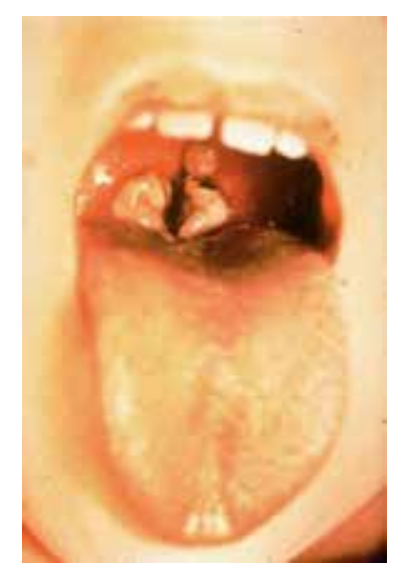

Figure 15. Diphtheria in the pharynx/Centers for Disease Control and Prevention/

Angina caused by listeria spp. is commonly seen among children between 7-18 years old. The disease starts acutely, sore throat and angina develop. The tonsils are covered by pus coating. In some cas- 
Iliyan Todorov, Dimitrichka Bliznakova, Christiana Madjova et al.

es regional lymph nodes are involved in the process. They are enlarged and painless. Most often, epidemiologic data about previous drinking of raw milk are accessible $(7,10)$.

Tularemia or rat-bite fever leads to the typical changes in the throat, affecting one of the tonsils most often. The epidemiologic data give information about preceding consummation of rabbit meat or food/water contaminated by rodents' urine. In some cases regional lymph nodes are also involved in the granulomatous type of inflammation. Patients' complaints are fever, asthenia, myalgia, intoxication, sore throat and harmed swallowing. Most often the changes in the oral cavity are unilateral - there is hyperemic and enlarged tonsil covered by white or greyish thick coating. Soon the coating falls down and necrosis appears on the tonsil surface. The prognosis is auspicious if the treatment starts on time.

A clinical polymorphism is typical about adenoviral infections but some strains cause changes in the oral mucosa in the throat predominantly. In the most cases, hyperemia and swelling of the pharynx appear which is granulated because of the hyperplasia of the lymph nodes. Follicular angina rarely may be seen. Sometimes pharyngitis is associated with conjunctivitis on the same side - pharyngoconjunctival fever /Parinaud's syndrome/ $(7,10,13,15)$.

Pharingitis is an early symptom of hemorrhagic fever Lassa. The disease starts after eating food, drinking water or inhalation powder contaminated by rodents' stools. Another mode of transmission includes direct contact with infected persons' blood. The disease is distributed in the village regions of West Africa, Burkina Faso and Zair. After acute onset fever, abdominal pain, nausea, vomiting and diarrhea occur. Sore throat and hard swallowing are characteristic. The pharynx is redness coatings on the tonsils and soft palate appear in association with regional lymph nodes enlargement. Hemorrhagic syndrome is mild, but in spite of this the mortality rate is high.

Infectious mononucleosis is acute infectious disease in the childhood caused by EBV. The beginning of the disease includes symptoms like fever, sore throat and cervical lymphadenitis. The oral changes are considerable: hypertrophic tonsils covered by pus follicular or lacunar coating (figure 16). The coat- ing is localized on the tonsils surface only its color is white and may easy be removed without revealing of bloody mucosa. It is the most important feature distinguishing infectious mononucleosis from diphtheria. After the first day of the disease enlargement of axillary lymph nodes, liver and spleen develop. In $10-15 \%$ of the cases scarlatiniform or maculopapular rashes appear.

The inspection of the oral cavity must not be missed as a part of the medical examination of the patient with infectious diseases. The physician has to know the condition of gingival mucosa, tongue,



Figure 16. Infectious mononucleosis /Color Atlas \& Synopsis of Pediatric Dermatology Kay Shou-Mei Kane, Jen Bissonette Ryder, Richard Allen Johnson, Howard P. Baden, Alexander Stratigos/

pharynx, uvula, tonsils, palatoglossal arches and buccal surface. It is more difficult in the childhood because of the low compliance from the patients' side. But oral cavity changes are pathognomonic for some diseases, facultative in other diseases, but when are associated with the main sign and symptoms aid to right diagnosis. The local changes must be commented in combination with the general features of the disease. It is the way for physicians to diagnosis. The opportunely and exact diagnosis in infectious diseases is of great significance because of their infectiousness and necessity for patients' isolation to prevent endemic situation.

Photographs No: 1-3,5,7,11,12,14-16 belong to the authors.

Photographs No: 4, 6, 8-10, 13 are copied from articles with open internet access. 


\section{REFERENCES}

1. Bliznakova B. Pediatrics for dentists. In: Diseases of the lips and oral mucosa. Varna; 2010. 395-412. Bulgarian.

2. D Genov, Lahtariev St, Stoicheva M, Komitova $P$, Geneva M. Changes in the mouth and throat. In: Symptoms and syndromes of infectious diseases. Sofia; 2007. 57-66. Bulgarian.

3. Dikov I, Zlatkov H, HIV-infection and AIDS in children. In: HIV-infection and AIDS. Plovdiv. 4648. Bulgarian.

4. Taskov B. Diphtheria of the throat. Diphtheria. Plovdiv; 1960. 40-55.

5. Alcaide ML, Bison AL: Pharyngitis and epiglottitis. Infect Dis Clin North Am.2007;21:449-469.

6. Bisno AL. Acute pharyngitis. N Engl J Med. 2001 Jan;344(3):205-11.

7. Bisno AL, Gerber MA, Gwaltney JM Jr, et al: Practice guidelines for the diagnosis and management of group A streptococcal pharyngitis. Clin Infect Dis. 2002;35:113-25.

8. Braunwald E, Fauci AS, Kasper DL, et al., eds. Harrison's Principles of Internal Medicine. 15th ed. New York, NY: McGraw-Hill; 2001.

9. Cunha BA. Group A streptococcal pharyngitis. Emerg Med. 1990;22:93-96.

10. Gerber MA. Diagnosis and treatment of pharyngitis in children. Pediatr Clin North Am. Jun 2005;52(3):729-47.

11. Gwaltney JM Jr, Bisno AL. Pharyngitis. In: Mandell GI, Bennett JE, Dolin R, eds. Mandell, Douglas \& Bennett's Principles of Infectious Diseases. Vol 1. Churchill Livingstone; 2000:656-62.

12. McMillan JA, Weiner LB, Higgins AM, Lamparella VJ: Pharyngitis associated with herpes simplex virus in college students. Pediatr Infect Dis. 1993;12:280-4.

13. Paradise JL. Etiology and management of pharyngitis and pharyngotonsillitis in children: a current review.Ann Otol Rhinol Laryngol Suppl. 1992 Jan;155:51-7.

14. Patel NN, Patel DN. Acute exudative tonsillitis. Am J Med. Jan 2009;122(1):18-20.

15. Perkins A. An approach to diagnosing the acute sore throat. Am Fam Physician. 1997 Jan;55(1):131$8,141-2$. 